\title{
BMJ open Microbleeds as a predictor of intracerebral haemorrhage and ischaemic stroke after a TIA or minor ischaemic stroke: a cohort study
}

\author{
Vincent I H Kwa, ${ }^{1}$ Ale Algra, ${ }^{2,3}$ Manon Brundel, ${ }^{2}$ Willem Bouvy, ${ }^{2}$ L Jaap Kappelle, ${ }^{2}$ \\ on behalf of the MICRO Study Group
}

To cite: Kwa VIH, Algra A, Brundel $\mathrm{M}$, et al. Microbleeds as a predictor of intracerebral haemorrhage and ischaemic stroke after a TIA or minor ischaemic stroke: a cohort study. BMJ Open 2013;3: e002575. doi:10.1136/ bmjopen-2013-002575

- Prepublication history and additional material for this paper is available online. To view these files please visit the journal online (http://dx.doi.org/10.1136/ bmjopen-2013-002575).

Received 8 January 2013

Revised 2 April 2013

Accepted 22 April 2013

This final article is available for use under the terms of the Creative Commons Attribution Non-Commercial 2.0 Licence; see http://bmjopen.bmj.com

${ }^{1}$ Department of Neurology, Onze Lieve Vrouwe Gasthuis, Slotervaart Hospital, Amsterdam, The Netherlands ${ }^{2}$ Department of Neurology and Neurosurgery, UMC Utrecht Stroke Centre, University Medical Centre Utrecht, Utrecht, The Netherlands

${ }^{3}$ Julius Centre for Health Sciences and Primary Care, Utrecht, The Netherlands

Correspondence to Dr Vincent I H Kwa; v.i.h.kwa@olvg.nl

\section{ABSTRACT}

Objectives: We examined whether patients with cerebral microbleeds on MRI, who started and continued antithrombotic medication for years, have an increased risk of symptomatic intracerebral haemorrhage (ICH).

Design: Prospective cohort study.

Settings: Multicentre outpatient clinics in the Netherlands.

Participants: We followed 397 patients with newly diagnosed transient ischaemic attack (TIA) or minor ischaemic stroke receiving anticoagulants or antiplatelet drugs. $58 \%$ were men. The mean age was 65.3 years $395(99 \%)$ patients were white Europeans. MRI including a T2* ${ }^{*}$-weighted gradient echo was performed within 3 months after start of medication. $48(12 \%)$ patients had one or more microbleeds. They were followed every 6 months by telephone for a mean of 3.8 years.

\section{Primary and secondary outcome measures:} Primary outcome was a symptomatic ICH. Secondary outcome were all strokes, ischaemic stroke, myocardial infarct, death from all vascular causes, death from nonvascular causes and death from all causes.

Results: Five patients (1\%) suffered from a symptomatic $\mathrm{ICH}$. One ICH occurred in a patient with microbleeds at baseline (adjusted HR 2.6, 95\% $\mathrm{Cl} 0.3$ to 27). The incidence of all strokes during follow-up was higher in patients with than without microbleeds (adjusted HR 2.3, $95 \% \mathrm{Cl} 1.0$ to 5.3), with a dose-response relationship. The incidences of ischaemic stroke, vascular death, non-vascular death and death of all causes were higher in patients with microbleeds, but not statistically significant

Conclusions: In our cohort of patients using antithrombotic drugs after a TIA or minor ischaemic stroke, we found that microbleeds on MRI are associated with an increased risk of future stroke in general, but we did not find an increased risk of symptomatic $\mathrm{ICH}$.

\section{INTRODUCTION}

Secondary prophylaxis with antithrombotics in patients with a transient ischaemic attack (TIA) or ischaemic stroke caused by

\section{ARTICLE SUMMARY}

Article focus

- Use of antithrombotic medication is associated with increased risk of intracerebral haemorrhage (ICH).

- Patients who use antithrombotic medication and have cerebral microbleeds on MRI may have an increased risk of $\mathrm{ICH}$ compared with patients without cerebral microbleeds.

- This finding is consistent with those in Asian studies of patients with microbleeds, but European studies showed conflicting results.

Key messages

- In our cohort of mainly white European patients using antithrombotic drugs after a TIA or minor ischaemic stroke, we did not find an increased risk of symptomatic $\mathrm{ICH}$ in patients with microbleeds on MRI.

- We found that the number of microbleeds on $M R I$ in patients using antithrombotic drugs after a TIA or minor ischaemic stroke was associated with the risk of future stroke in general.

atherosclerosis is effective. ${ }^{1}$ In patients with non-valvular atrial fibrillation, oral anticoagulants are the first choice, whereas in patients with sinus rhythm, antiplatelet drugs should

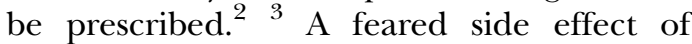
antithrombotics is an intracerebral haemorrhage (ICH), which has an annual incidence of $0.3-2 \%$, depending on the type of antithrombotic medication. ${ }^{4}$ In patients with no or minor residual deficits after the initial stroke, this issue is of particular importance. Although the incidence of ICH is low, the less residual symptoms there are after the initial stroke, the larger the impact is of new neurological deficit due to ICH caused by prophylactic medication.

Microbleeds are small hypointense dots on T2*-weighted gradient echo MRI. Probably, they represent the remnants of small 


\section{ARTICLE SUMMARY}

Strengths and limitations of this study

- The strength of this study is its specific design for following patients with microbleeds. The follow-up is nearly complete and is the longest in this type of studies until now. Owing to the multicentre design, the results can be extrapolated to a general neurology practice. The population of patients whom we studied is an important subgroup of stroke patients, since they had no or only minor neurological deficits after their event and had much to lose if they would have a complication of their prophylactic treatment.

- The cohort is representative of a contemporary outpatients vascular clinic with a comparable prevalence of microbleeds, proportion of patients using oral anticoagulants and overall risk for symptomatic ICH and ischaemic events, similar to that in prior recent studies with the same population of patients.

- A limitation of this study is that it is underpowered due to a lower incidence of ICH than we expected. Our estimate of the relationship between microbleeds and ICH might be considered as imprecise.

asymptomatic ICHs and are considered a sign of cerebral microangiopathy. ${ }^{5}$ They have been reported in $5 \%$ of the healthy elderly and in $10-15 \%$ of patients with cerebral infarcts. ${ }^{6}$

Cerebral microbleeds may be a marker of the tendency of the brain to bleed. Patients with these lesions have an increased risk for $\mathrm{ICH}^{6}{ }^{6}$ In patients with normal haemostasis, these spontaneous intracerebral bleedings are thought to remain small and asymptomatic. However, under the influence of antithrombotic drugs, they might grow into a larger symptomatic bleeding. This mechanism might be the explanation for the higher risk of ICH in Asian patients using antithrombotics and the unexpected high proportion of ICH in a previous trial with anticoagulants in patients with nondisabling cerebral ischaemia of arterial origin. ${ }^{7-10}$

We studied a prospectively collected cohort of European patients with no or minor residual deficits after the initial ischaemic stroke who were prescribed antithrombotic drugs, with the aim of calculating the risk for a future symptomatic ICH that is associated with the presence of microbleeds.

\section{SUBJECTS AND METHODS}

From June 2000 to January 2010, we prospectively included patients with a TIA or a minor ischaemic stroke (modified Rankin score of 3 or less) from 10 academic and non-academic hospitals in the Netherlands. This study started as a satellite study of the ESPRIT trial and continued on its own after completion of ESPRIT in December 2005. ${ }^{11}$ Patients had to be started on anticoagulants or antiplatelet drugs because of the TIA or minor stroke within the previous 3 months. We excluded patients who were expected to die within months, with a low ability to understand or express themselves in the
Dutch language, or those who were pregnant. We also excluded patients with a history of intracranial haemorrhage, brain concussion, or cerebral tumour and patients with a TIA or minor stroke caused by vasculitis. Follow-up was performed by a central trial office.

All patients underwent prespecified MRI including a T2*-weighted fast field echo (FFE) gradient echo within 3 months after the start of medication. We provided all centres with a protocol of MRI sequences (see online supplementary table S1). The MRI sequence parameters that were eventually used in most centres are provided in online supplementary table S2. Data and imaging were collected centrally. MRIs were read centrally by two independent investigators familiar with these imaging techniques. The presence of microbleeds was scored with the Microbleed Anatomical Rating Scale. ${ }^{12}$ The $\kappa$ of this rating was 0.36 , which is within the limits of 0.33 0.95 that have been published before in studies of microbleeds. ${ }^{6}$ White matter lesions were scored according to the Age-Related White Matter Changes scale. ${ }^{13}$

The institutional medical ethics review boards of the participating hospitals approved the study protocol, and all patients provided written informed consent.

Patients were followed up every 6 months by telephone. At each contact, the occurrence of possible outcome events and hospital admissions was recorded. In case an event occurred, a report on the clinical details was requested. For all strokes during follow-up, we required a confirmation by CT scan, MRI or autopsy to determine the ischaemic or haemorrhagic nature of the stroke. For subtyping the ischaemic strokes, we used the Oxford classification. ${ }^{14}$ Except for four patients who were lost to follow-up, all patients had a close-out contact between 1 February 2011 and 1 May 2011. All data were collected and checked at the central trial office and entered in a database. During the study, none of the investigators had any knowledge of the event rates according to the presence or absence of microbleeds.

The primary outcome event was an ICH. Secondary endpoints included all strokes, ischaemic stroke, myocardial infarct, death from all vascular causes, death from non-vascular causes and death from all causes.

Death from vascular causes included death caused by cerebral infarction, intracranial haemorrhage, unspecified stroke, myocardial infarction, heart failure, pulmonary embolism, arterial bleeding or sudden death. ${ }^{11}$ If no information was available about the cause of death, we classified the reason as vascular other, according to a priori probabilities. ${ }^{15}$ Non-fatal ischaemic stroke was diagnosed in case of sudden onset of a new or increasing neurological deficit that persisted for more than $24 \mathrm{~h}$, resulting in an increase in handicap of at least one grade on the modified Rankin scale, and no signs of haemorrhage on the CT scan or MRI of the brain made within 2 weeks of the event. We used the same clinical criteria for the diagnosis of haemorrhagic stroke if a corresponding ICH was detected on CT scan or MRI of the brain. If no brain imaging or autopsy was performed 
and clinical evidence of stroke was present, we classified the event as stroke, unspecified. The outcome event of myocardial infarction required at least two of the following characteristics: a history of chest discomfort for at least half an hour, level of specific cardiac enzymes more than twice the upper limit of normal or the development of specific abnormalities (eg, $Q$ waves) on the standard 12-lead ECG.

Outcome events were reported to the central trial office where all relevant data, including a brain scan or ECG, were obtained from the physician in charge. The clinical report of the outcome event was presented to two investigators (VIHK, LJK); they independently classified the event. If the classifications differed, the outcome event was discussed by the investigators, who made a decision by consensus.

\section{Statistical analysis}

We assumed a prevalence of microbleeds of $15 \%$ and an annual risk of ICH in patients on antiplatelet or anticoagulant drugs of $0.31 \% .{ }^{11}$ With 1800 patient-years, we would be able to detect a risk of symptomatic ICH with a risk ratio (RR) of 7.6 with a $95 \%$ CI of 1.3 to 52 .

We compared the risk of ICH between patients with and without microbleeds at baseline by means of Cox regression analysis. Resulting HRs were accompanied by corresponding 95\% CI. In addition to crude HRs, we calculated age-adjusted and sex-adjusted HRs.

\section{RESULTS}

We included 448 patients, of whom 51 did not have an FFE-gradient echo series during the MRI due to various reasons, leaving 397 analysable patients (figure 1). Patients who did not have an FFE-gradient echo MRI were, on average, 5 years younger, smoked more often ( $45 \%$ vs $27 \%$ ) and had hyperlipidaemia (20\% vs $42 \%$ ) or atrial fibrillation $(0 \%$ vs $8 \%$ ) less frequently than patients who had an FFE-gradient echo MRI. There were no other differences between the two groups concerning sex, ethnicity, Rankin scores and other vascular risk factors (see online supplementary table S3).

Patient characteristics of the 397 analysable patients are given in table 1. At inclusion, 101 patients (25.4\%) were using or had used antiplatelet agents some time in the past. One hundred and ninety-four $(48.9 \%)$ had a TIA, $21(5.3 \%)$ a transient monocular blindness and 183 $(45.8 \%)$ a minor stroke. $392(98.7 \%)$ had a Rankin score of 3 or less. MRI was performed within a median of 11 days (25th-75th centiles: 4-55 days) after the TIA or minor stroke. Of these patients, $19(4.8 \%)$ were scanned with a $0.5 \mathrm{~T}$ MRI, $71(17.9 \%)$ with a $1.0 \mathrm{~T}$ MRI and $307(77.3 \%)$ with a $1.5 \mathrm{~T}$ MRI. Although the number of patients scanned with a $0.5 \mathrm{~T}$ MRI was small, there were no microbleeds detected with this type of scans. The proportion of patients detected to have microbleeds with a 1.0 or $1.5 \mathrm{~T}$ MRI did not differ significantly ( $16.9 \%$ and $11.7 \%$, respectively).

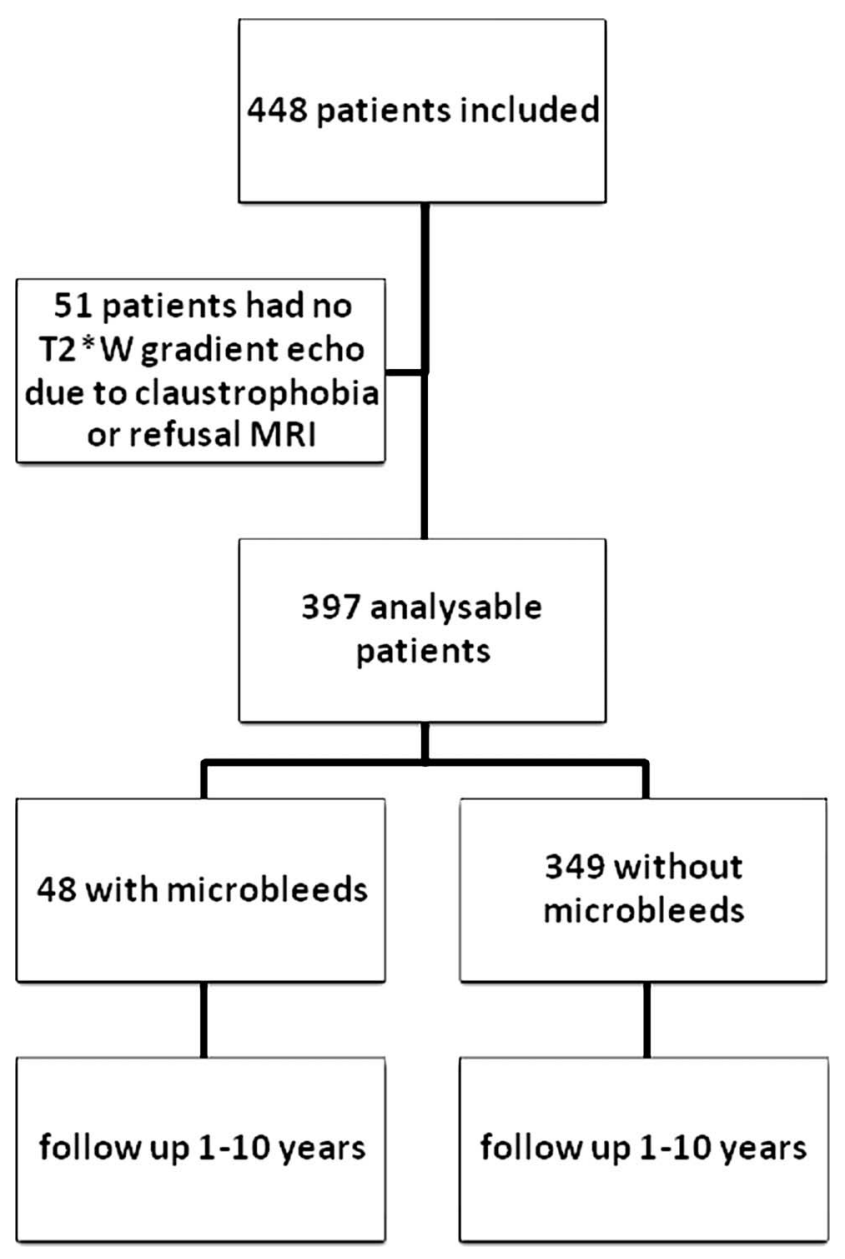

Figure 1 Flow chart of the study.

Forty-eight $(12 \%)$ patients had microbleeds, of which $29(7 \%)$ had one, $16(4 \%)$ had between 2 and 10, and 3 (1\%) had more than 10 microbleeds. Patients with microbleeds were older and had slightly worse Rankin scores. The mean duration of follow-up was 3.8 (SD 2.5) years, varying from 0.1 to 10.4 years, accumulating to 1509 patient-years. Four (1\%) patients were lost to follow-up. During follow-up, $40(10.1 \%)$ patients used oral anticoagulants, but all others used antiplatelet drugs. Data on adherence to the medication for patients who also participated in the ESPRIT trial were as follows: at 5 years, $66 \%$ patients were on aspirin plus dypiridamole, $84 \%$ on aspirin alone and $68 \%$ on oral anticoagulation. ${ }^{11}$

There were five $(1.3 \%$, overall incidence $0.3 \%$ /year (95\% CI 0.1 to $0.8 \% /$ year)) patients who had a symptomatic ICH during follow-up; of these, only one occurred in a patient with microbleeds at baseline. In this patient, we detected 5 microbleeds. The corresponding crude HR was 1.8 (95\% CI 0.2 to 16$)$ and 2.6 (95\% CI 0.3 to 27) after adjustment for age and sex (table 2).

The incidence of all strokes was higher in patients with microbleeds (crude HR 2.6, 95\% CI 1.1 to 6.2), also after correction for age and sex (HR 2.3, 95\% CI 1.0 to 5.3; figure 2). The more microbleeds a patient 
Table 1 Patients' baseline characteristics of the MRI cohort

\begin{tabular}{lcc}
\hline & With $\mathbf{M B}, \mathbf{n = 4 8}$ & Without MB, $\mathbf{n = 3 4 9}$ \\
\hline Mean age (SD) & $70.8(8.8)$ year & $64.6(12.4)$ year \\
Men & $28(58 \%)$ & $204(59 \%)$ \\
White European ethnicity & $48(100 \%)$ & $347(99 \%)$ \\
Rankin score & & $159(46 \%)$ \\
0 & $15(31 \%)$ & $107(31 \%)$ \\
1 & $18(38 \%)$ & $83(24 \%)$ \\
$\geq 2$ & $15(31 \%)$ & $185(53 \%)$ \\
Hypertension & $33(69 \%)$ & $152(29) \mathrm{mm} \mathrm{Hg}$ \\
$\quad$ Mean (SD) systolic blood pressure & $164(35) \mathrm{mm} \mathrm{Hg}$ & $86(16) \mathrm{mm} \mathrm{Hg}$ \\
$\quad$ Mean (SD) diastolic blood pressure & $86(14) \mathrm{mm} \mathrm{Hg}$ & $47(14 \%)$ \\
Diabetes mellitus & $7(15 \%)$ & $95(27 \%)$ \\
Smoking & $12(25 \%)$ & $144(41 \%)$ \\
Hyperlipidaemia & $21(44 \%)$ & $20(6 \%)$ \\
Myocardial infarct & $4(8 \%)$ & $10(3 \%)$ \\
Intermittent claudication & $2(4 \%)$ & $28(8 \%)$ \\
Atrial fibrillation & $2(4 \%)$ & $28(8 \%)$ \\
Previous ischaemic stroke & $7(15 \%)$ & \\
\hline MB, microbleeds; numbers (\%), unless stated otherwise. & & \\
\end{tabular}

had, the higher was the risk for future strokes (table 3). The incidence of ischaemic strokes was also higher in patients with microbleeds, but the HRs did not reach statistical significance. Interestingly, of the ischaemic strokes in patients with microbleeds, the proportion of lacunar infarcts $(67 \%)$ was larger than that in patients without microbleeds $(35 \%)$, but again this was not statistically significant (see online supplementary table S4). The incidence of myocardial infarction was low and did not occur at all in patients with microbleeds. Vascular deaths, non-vascular deaths and deaths of all causes were also more frequent in patients with microbleeds, but did not reach statistical significance.

Since white matter lesions are strongly associated with microbleeds, an additional analysis with white matter lesions was performed, but white matter lesions were not associated either with a higher risk of future ICH or ischaemic stroke (data not shown).

\section{DISCUSSION}

In our cohort, we did not observe an increased risk for future symptomatic ICHs in patients with microbleeds who used antithrombotic drugs after a TIA or minor ischaemic stroke. However, microbleeds were associated with an increased risk of future stroke in general, with a dose-response relationship: the higher the number of microbleeds, the higher the risk of stroke. These findings confirm that microbleeds should be considered as a biomarker that is associated with an increased risk of future cerebral vascular events. We included patients with no or minor deficits, because in these patients new neurological deficits would have a larger impact than in patients with severe residual deficit caused by the initial stroke.

Our cohort is representative of a contemporary outpatients vascular clinic. The low overall risk for symptomatic ICH and ischaemic events resembles that of prior recent studies in patients on antithrombotic agents for secondary prevention and concurs with our assumptions in the power calculation. ${ }^{10} 16$ The prevalence of microbleeds was similar to that reported in patients with a TIA or ischaemic stroke. ${ }^{6}$ Moreover, the proportion of patients who used oral anticoagulants equals that in other studies. $^{16}$

Table 2 Outcome events

\begin{tabular}{|c|c|c|c|c|}
\hline & With MB & Without MB & Crude HR $(95 \% \mathrm{Cl})$ & $\begin{array}{l}\text { HR adjusted for age and } \\
\operatorname{sex}(95 \% \mathrm{Cl})\end{array}$ \\
\hline $\mathrm{ICH}$ & $1(2.1 \%)$ & $4(1.1 \%)$ & 1.8 (0.2 to 16$)$ & 2.6 (0.3 to 27$)$ \\
\hline Ischaemic stroke & $6(12.5 \%)$ & $17(4.9 \%)$ & 2.7 (1.1 to 6.9$)$ & 2.3 (0.9 to 5.8$)$ \\
\hline All strokes & $7(14.6 \%)$ & $21(5.7 \%)$ & 2.6 (1.1 to 6.2$)$ & 2.3 (1.0 to 5.3 ) \\
\hline Subdural bleeds & $1(2.1 \%)$ & $5(1.4 \%)$ & $1.3(0.2$ to 12$)$ & $1.0(0.1$ to 8.9$)$ \\
\hline Myocardial infarct & $0(0 \%)$ & $4(1.1 \%)$ & Not estimable & Not estimable \\
\hline Vascular death & $5(10.4 \%)$ & $16(4.6 \%)$ & $2.2(0.8$ to 6.0$)$ & 1.7 (0.6 to 4.8$)$ \\
\hline Non-vascular death & $4(8.3 \%)$ & 15 (4.3\%) & $1.8(0.6$ to 5.5$)$ & 1.4 (0.5 to 4.3$)$ \\
\hline All deaths & $9(18.8 \%)$ & $31(8.9 \%)$ & 2.0 (1.0 to 4.2$)$ & 1.6 (0.8 to 3.3$)$ \\
\hline
\end{tabular}




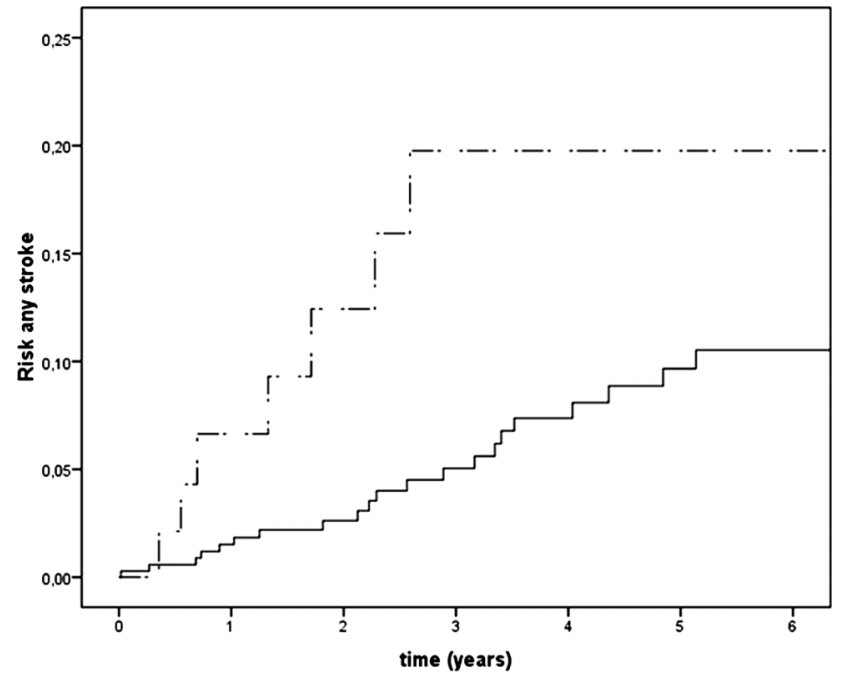

Figure 2 Cumulative number of any kind of strokes in patients with microbleeds (dotted line) or without microbleeds (straight line).

The risk for ICH in stroke patients treated with antithrombotic agents after an ischaemic stroke probably differs between Eastern and Western patients. In two Chinese studies, a higher risk of ICH was found in patients with microbleeds with HRs of 7.95 and 7.3, respectively, but with large CI $(95 \%$ CI, 2.56 to 24.66 , and 0.8 to 63 , respectively). ${ }^{78}$ A Japanese study reported an RR of 50 (95\% CI 16.7 to 150.9). ${ }^{9}$ In a European hospital-based cohort of patients with a major stroke, a trend to a higher risk of ICH was observed in patients with than in patients without microbleeds (adjusted HR 1.6, $95 \%$ CI 0.8 to 3.1$).{ }^{17}$ In a Canadian cohort of stroke patients, only one ICH occurred in both group of patients with and without MB. ${ }^{18}$ In a large European case-control study of patients with ICH, a higher risk of a new ICH during the use of antiplatelet drugs was observed in patients with than in patients without microbleeds (OR $1.27,95 \%$ CI 1.04 to 1.55$).{ }^{19}$ In these publications, the population studied was either of a different ethnicity or had another type of cerebrovascular disease than our population with inherently different baseline risks of ICH. A systematic review of published and unpublished cohorts, including a small European prospective cohort of ischaemic stroke or TIA patients using antithrombotic drugs, suggested that anticoagulants may be hazardous in patients with microbleeds. ${ }^{20}$

The strength of our study is its specific design for the following patients with microbleeds with a nearly complete and the longest follow-up until now. Ninety-nine per cent of patients could be followed up until the close-out visit. Second, owing to the multicentre design, the results can be extrapolated to a general neurology practice. Third, we think that the population of patients we studied is an important subgroup of stroke patients, since they had no or only minor neurological deficits after their event and had much to lose if they would have a complication of their prophylactic treatment.

There are several limitations to our study. Since the incidence of ICH was lower than we expected, the study is underpowered and our estimate of the relationship between microbleeds and ICH might be considered as imprecise. To reach a more accurate result, many more patient-years would be needed. A formal meta-analysis of all available studies might solve this problem. However, even when the risk turns out to be higher in patients with microbleeds, the absolute numbers of ICH are so low that, in our opinion, the efforts to screen patients for microbleeds to prevent a single ICH would not be cost-effective compared with the benefits of antithrombotic drugs to prevent ischaemic events. Our results do not support the need for MRI scanning in every patient in whom antithrombotic treatment should be prescribed.

An explanation for the low incidence of ICH is that most of our patients used aspirin instead of oral anticoagulants. The incidence of ICH was similar to that in studies on patients on antiplatelet drugs. For the few patients on oral anticoagulants, we cannot exclude that the presence of microbleeds predicts a higher risk of ICH. Further studies with European cohorts of patients using anticoagulants in the presence of microbleeds are underway.

A biological explanation for the low incidence of ICH is that the effects of microangiopathy in a white European population differ from those in an Eastern population. The incidence of ICH in Eastern populations is twice as high as in white Western people. ${ }^{21}$ The interaction between genetic variations with environmental factors differs in Asian and Western patients. ${ }^{21}$ For example, Asian carriers of the APOE genotype $\varepsilon 2$ and $\varepsilon 4$ have almost twice as large a risk of future ICH compared with European carriers. ${ }^{22}$

A third possible explanation for the low incidence of $\mathrm{ICH}$ is that the presumed microhaemorrhages are in fact no small ICHs caused by breaking of the small vessels, but merely leakage of the blood-brain barrier, a

Table 3 Risk for any stroke according to the number of microbleeds

\begin{tabular}{lllll}
\hline Number of microbleeds & Number of patients & $\begin{array}{l}\text { Number of patients } \\
\text { with any stroke }\end{array}$ & $\begin{array}{l}\text { Crude HR } \\
(\mathbf{9 5 \%} \mathbf{C l})\end{array}$ & $\begin{array}{l}\text { HR adjusted for age and } \\
\text { sex (95\% Cl) }\end{array}$ \\
\hline 0 & 349 & 21 & ref & ref \\
1 & 29 & 3 & $1.8(0.5$ to 6.1) & $1.5(0.5$ to 5.2$)$ \\
$>1$ & 19 & 4 & $3.9(1.3$ to 11.4) & $3.5(1.2$ to 10) \\
\hline
\end{tabular}


hypothesis in which breakdown of the blood-brain barrier would lead to both lacunar infarcts and microbleeds, as well as white matter lesions. ${ }^{23}$ Such a hypothesis might explain the higher risk for both ICH and ischaemic strokes in patients with microbleeds, since microbleeds are in that case also a marker of the underlying disease leading to both ICH and lacunar infarcts.

An important portion (40\%) of our patients with microbleeds only had one microbleed. Radiologicalpathological correlations of microbleeds are scarce. Although we excluded microbleed-mimics, a single microbleed might not be a sign of general microangiopathy. Also, not all centres had high quality MRI's at the time of the study. Although a minority, some patients were scanned with a $0.5 \mathrm{~T}$ MRI, and in none of them did we find a microbleed. Therefore, we might have underestimated the number of microbleeds. ${ }^{24}$ With newer high-field MR-techniques, more microbleeds in more patients can be detected, perhaps revealing true microangiopathy. ${ }^{25}$

In conclusion, we have demonstrated that in our cohort of mainly white European patients the number of microbleeds on gradient echo MRI is associated with the risk of future stroke in general, but we could not demonstrate an association of microbleeds with future symptomatic ICH. There is no need to withhold antiplatelet agents in these patients. The number of patients who used anticoagulants in this study was too small to draw definitive conclusions in this perspective.

Acknowledgements We thank Mrs Paut Greebe, Mrs Dorien CV Slabbers, Mrs Ans de Ridder, Mrs Saskia Scott, Mrs Olga A Ternede, Mrs Moniek de Rijk, Mrs Sigrid van Steen, Mrs Jasmijn Y Lodico-Kuipers, Mrs Sandra Kleijn, Mrs Hanneke M Droste, Mrs Barbara F Drijber, Mrs Helen Blad, Mrs Nadine Fleitour and Mrs Anick Gorissen for collecting the data; Geert-Jan Biessels, neurologist, for critically appraising the data and classifying the events. Participating centres MICRO (Microbleeds on MRI as predictors of IntraCerebral haemorrhage in patients Receiving Oral antithrombotic drugs after TIA or a minor ischaemic stroke) Study Group (with the numbers of patients included and investigators): University Medical Centre, Utrecht (256, A Algra, LJ Kappelle, MP Ramos,

ELLM De Schryver); Slotervaart Hospital Amsterdam (107, VIH Kwa, GJ Jöbsis, JJ van der Sande); Medisch Spectrum Twente, Enschede (29, PJAM Brouwers); Academic Medical Centre, Amsterdam (17, YEBM Roos, J Stam); Amphia Hospital, Breda (15, SLM Bakker, HBC Verbiest); St Antonius Hospital Nieuwegein (11, WJ Schoonewille); Central Military Hospital, Utrecht (5, FHH Linn); Albert Schweitzer Hospital, Dordrecht (3, LI Hertzberger); Meander Medical Centre, Amersfoort (3, HMA van Gemert); Lievensberg Hospital, Bergen op Zoom (2, PJIM Berntsen).

Contributors VIHK and LJK had the idea of the study. VIHK, AA and LJK designed the study. VIHK wrote the first draft of the manuscript. MB and WB read the MRI's and contributed to the collection of the data. VIHK and AA did the statistical analysis. All authors contributed to the writing of the article and approved the final version.

Funding This work was supported by the Netherlands Heart Foundation (grant 2001B071).

Competing interests None.

Ethics approval The study protocol was approved by the institutional medical ethics review boards of the participating hospitals. All patients provided written informed consent.

Provenance and peer review Not commissioned; externally peer reviewed.

Data sharing statement No additional data are available.

\section{REFERENCES}

1. Antithrombotic Trialists' Collaboration. Collaborative meta-analysis of randomised trials of antiplatelet therapy for prevention of death, myocardial infarction, and stroke in high risk patients. BMJ 2002;324:71-86.

2. The European Stroke Organisation (ESO) Executive Committee and the ESO Writing Committee. Guidelines for Management of Ischaemic Stroke and Transient Ischaemic Attack 2008. Cerebrovasc Dis 2008;25:457-507.

3. Furie KL, Kasner SE, Adams RJ, et al. Guidelines for the prevention of stroke in patients with stroke or transient ischemic attack. Stroke 2011;42:227-76.

4. Hart RG, Tonarelli SB, Pearce LA. Avoiding central nervous system bleeding during antithrombotic therapy. Stroke 2005;36:1588-93.

5. Fazekas F, Kleinert R, Roob G, et al. Histopathologic analysis of foci of signal loss on gradient-echo T2*-weighted MR images in patients with spontaneous intracerebral hemorrhage: evidence of microangiopathy-related microbleeds. AJNR 1999;20:637-42.

6. Cordonnier C, Al-Shahi Salman R, Wardlaw J. Spontaneous brain microbleeds: systematic review, subgroup analyses and standards for study design and reporting. Brain 2008;130:1988-2003.

7. Fan YH, Zhang L, Lam WWM, et al. Cerebral microbleeds as a risk factor for subsequent intracerebral hemorrhages among patients with acute ischemic stroke. Stroke 2003;34:2459-62.

8. Soo Y, Yang S, Lam W, et al. Risk vs benefit of anti-thrombotic therapy in ischaemic stroke patients with cerebral microbleeds. $J$ Neurol 2008;255:1679-86.

9. Bokura H, Saika R, Yamaguchi T, et al. Microbleeds are associated with subsequent hemorrhagic and ischemic stroke in healthy elderly individuals. Stroke 2011;42:1867-71.

10. The Stroke Prevention In Reversible Ischemia Trial (SPIRIT) Study Group. A randomized trial of anticoagulants versus aspirin after cerebral ischemia of presumed arterial origin. Ann Neurol 1997;42:857-65.

11. The ESPRIT Study Group. Aspirin plus dipyridamole versus aspirin alone after cerebral ischaemia of arterial origin (ESPRIT): randomised controlled trial. Lancet 2006;367:1665-73.

12. Gregoire SM, Chaudhary UJ, Brown MM, et al. The microbleed anatomical rating scale (MARS). Reliability of a tool to map brain microbleeds. Neurology 2009;73:1759-66.

13. Wahlund LO, Barkhof F, Fazekas F, et al. A new rating scale for age-related white matter changes. Applicable to MRI and CT. Stroke 2001;32:1318-22.

14. Bamford J, Sandercock $P$, Dennis $M$, et al. Classification and natural history of clinically identifiable subtypes of cerebral infarction. Lancet 1991;337:1521-6.

15. CAPRIE Steering Committee. A randomised, blinded, trial of clopidogrel versus aspirin in patients at risk of ischaemic events (CAPRIE). Lancet 1996;348:1329-39.

16. Lavallée $\mathrm{PC}$, Meseguer $\mathrm{E}, \mathrm{Abboud} \mathrm{H}$, et al. A transient ischaemic attack clinic with round-the-clock access (SOS-TIA): feasibility and effects. Lancet Neurol 2007;6:953-60.

17. Thijs V, Lemmens R, Schoofs C, et al. Microbleeds and the risk of recurrent stroke. Stroke 2010;41:2005-9.

18. Boulanger JM, Coutts SB, Eliasziw M, et al. Cerebral microhemorrhages predict new disabling or fatal strokes in patients with acute ischemic stroke or transient ischemic attack. Stroke 2006;37:911-14.

19. Gregoire SM, Jäger HR, Yousry TA, et al. Brain microbleeds as a potential risk factor for antiplatelet-related intracerebral haemorrhage: hospital-based, case-control study. J Neurol Neurosurg Psychiatry 2010;81:679-84.

20. Lovelock CE, Cordonnier $\mathrm{C}$, Naka H, et al. Antithrombotic drug use, cerebral microbleeds, and intracerebral hemorrhage. Stroke 2010;41:1222-8.

21. van Asch CJ, Luitse MJ, Rinkel GJ, et al. Incidence, case fatality, and functional outcome of intracerebral haemorrhage over time, according to age, sex, and ethnic origin: a systematic review and meta-analysis. Lancet Neurol 2010;9:167-76.

22. Tzourio $\mathrm{C}$, Arima $\mathrm{H}$, Harrap $\mathrm{S}$, et al. APOE genotype, ethnicity, and the risk of cerebral hemorrhage. Neurology 2008;70:1322-8.

23. Wardlaw JM, Sandercock PAG, Dennis MS, et al. Is breakdown of the blood-brain barrier responsible for lacunar stroke, leukoaraisosis, and dementia? Stroke 2003;34:806-12.

24. Gregoire SM, Werring DJ, Chaudhary UJ, et al. Choice of echo time on GRE T2*-weighted MRI influences the classification of brain microbleeds. Clin Radiol 2010;65:391-4.

25. Vernooij MW, Ikram MA, Wielopolski PA, et al. Cerebral microbleeds: accelerated 3D T2*-weighted GRE MR imaging versus conventional 2D T2*-weighted GRE MR imaging for detection. Radiology 2008;248:272-7. 